\title{
The enigmatic mitochondrial genome of Rhabdopleura compacta (Pterobranchia) reveals insights into selection of an efficient tRNA system and supports monophyly of Ambulacraria
}

Marleen Perseke ${ }^{1,2}$, Joerg Hetmank ${ }^{1}$, Matthias Bernt ${ }^{3}$, Peter F Stadler ${ }^{4,5,6,7,8,9,10^{*}}$, Martin Schlegel ${ }^{1,5}$ and Detlef Bernhard'

\begin{abstract}
Background: The Hemichordata comprises solitary-living Enteropneusta and colonial-living Pterobranchia, sharing morphological features with both Chordata and Echinodermata. Despite their key role for understanding deuterostome evolution, hemichordate phylogeny is controversial and only few molecular data are available for phylogenetic analysis. Furthermore, mitochondrial sequences are completely lacking for pterobranchs. Therefore, we determined and analyzed the complete mitochondrial genome of the pterobranch Rhabdopleura compacta to elucidate deuterostome evolution. Thereby, we also gained important insights in mitochondrial tRNA evolution.

Results: The mitochondrial DNA of Rhabdopleura compacta corresponds in size and gene content to typical mitochondrial genomes of metazoans, but shows the strongest known strand-specific mutational bias in the nucleotide composition among deuterostomes with a very GT-rich main-coding strand. The order of the proteincoding genes in $R$. compacta is similar to that of the deuterostome ground pattern. However, the protein-coding genes have been highly affected by a strand-specific mutational pressure showing unusual codon frequency and amino acid composition. This composition caused extremely long branches in phylogenetic analyses. The unusual codon frequency points to a selection pressure on the tRNA translation system to codon-anticodon sequences of highest versatility instead of showing adaptations in anticodon sequences to the most frequent codons. Furthermore, an assignment of the codon AGG to Lysine has been detected in the mitochondrial genome of $R$. compacta, which is otherwise observed only in the mitogenomes of some arthropods. The genomes of these arthropods do not have such a strong strand-specific bias as found in $R$. compacta but possess an identical mutation in the anticodon sequence of the tRNA $A_{\text {Lys. }}$.
\end{abstract}

Conclusion: A strong reversed asymmetrical mutational constraint in the mitochondrial genome of Rhabdopleura compacta may have arisen by an inversion of the replication direction and adaptation to this bias in the protein sequences leading to an enigmatic mitochondrial genome. Although, phylogenetic analyses of protein coding sequences are hampered, features of the tRNA system of $R$. compacta support the monophyly of Ambulacraria. The identical reassignment of AGG to Lysine in two distinct groups may have occurred by convergent evolution in the anticodon sequence of the tRNA $\mathrm{Lyy}_{\text {. }}$.

Keywords: Hemichordata, Pterobranchia, deuterostome evolution, codon reassignment, codon-anticodon adaptation

\footnotetext{
* Correspondence: studla@bioinf.uni-leipzig.de

${ }^{4}$ Bioinformatics Group, Dept. of Computer Science, University of Leipzig,

Härtelstr, 16-18, 04107 Leipzig, Germany

Full list of author information is available at the end of the article
}

(C) 2011 Perseke et al; licensee BioMed Central Ltd. This is an Open Access article distributed under the terms of the Creative Commons 


\section{Background}

Pterobranchia are a small, specialised group of marine suspension-feeding animals. Despite rich fossil record, only some 20 extant species have been described, which are subdivided into three genera, Rhabdopleura, Cephalodiscus and Atubaria [1]. One questionable species from the genus Atubaria (Atubaria heterolopha) is described, which lives solitary [2]. Species of Rhabdopleura and Cephalodiscus live in secreted tubes in colonies [1]. Although Pterobranchia comprise only few extant species, they represent an important deuterostome lineage. Usually they are grouped to the solitary-living Enteropneusta, forming together the Hemichordata (e.g. [3]). Because the Hemichordata unite features of the remaining two major deuterostome subgroups, Chordata and Echinodermata, the phylogenetic position of Pterobranchia is crucial for understanding the evolution of chordate and deuterostome body plans.

Despite their evolutionary importance, only ribosomal sequence data were analysed for phylogenetic reconstruction of the pterobranchs [4-6]. These analyses support the monophyly of Hemichordata and a close relationship with Echinodermata, forming the Ambulacraria [7]. However, results within this group are conflicting. The $28 S$ rDNA sequence analyses suggest reciprocal monophyly of Enteropneusta and Pterobranchia while the analyses of the $18 S \mathrm{rDNA}$ sequences support a branching of Pterobranchia within Enteropneusta.

A frequently used molecular marker for reconstruction of the phylogenetic relationships is the complete mitochondrial genome (e.g. [8-11]). The typical metazoan mitochondrial genome is a circular DNA molecule ranging from 15 to $20 \mathrm{~kb}$ in size and comprises a more or less conserved gene content of 13 protein-coding genes, two ribosomal RNA (rRNA) genes and 22 transfer RNA (tRNA) genes [12]. The complete genomes allow phylogenetic analyses of both sequences and gene order. The complete mitochondrial genomes are well characterised from Vertebrata (e.g. [12]), Cephalochordata [9], Tunicata (e.g. [13]), and the five recent echinoderm subgroups (e.g. [11]). The mitochondrial genome was also determined for the enigmatic worm-like Xenoturbella bocki supporting a close relationship to Deuterostomia [10,14]. With the exception of the highly derived Tunicata, mitochondrial genomes of Deuterostomia are similar in architecture [8]. However, variability has been found in the genetic code caused by different assignments of four codons (AGA, AGG, AAA, and AUA). Since the metazoan mitochondrial translation system comprises a reduced tRNA gene set of usually 22 tRNA genes, each tRNA gene has to recognize different codons (usually two or four codons), which often differ only in the $3^{\text {rd }}$ codon site. The influence of the $3^{\text {rd }}$ codon site on the translation efficiency is still under discussion [15-22].
The "theory of anticodon sequences with highest versatility" postulates that the anticodon sequence of a tRNA gene pairs equally effectively with all recognized codons $[15,16,18]$. Alternatively, the "theory of codon-anticodon adaptation" suggests that the anticodon sequence of a tRNA gene is adapted to the most frequent codon among all the recognized codons $[19,20]$. As most metazoan mitochondrial genomes show an AT-rich main-coding strand [23], the tRNAs preferentially have GNN anticodons for NNY codons, UNN for NNR codons, and also UNN for the four-fold degenerate codon families [19,20]. This is in congruence with both theories (see [17]). The strand-specific nucleotide bias may have arisen by the asymmetrical replication of the circular mitochondrial genome which exposes stretches of a DNA strand for a longer time as single-stranded than other parts, accumulating Adenine and Guanine nucleotides more faster [23-28]. However, some mitochondrial genomes possess an inverse strand-specific mutational pattern showing a GT-rich main-coding strand, e.g. Branchiostomia (Cephalochordata) and Crinoidea (Echinodermata), which is usually explained by an inversion of the replication direction of the mtDNA [23,29].

In contrast to these well characterized genomes, only three enteropneust genomes are known from Hemichordata. The two Balanoglossus genomes exhibit similarities to Vertebrata and Echinodermata [30]. The analyses of the genetic code suggest assignments as found in Echinodermata, although two important codons are missing (AAA and AAG) [31]. The genome of the enteropneust Saccoglossus kowalevskii was not described in detail, but the published annotated sequence shows a derived gene order and a different assignment of the codon AAA compared to the echinoderm genetic code (AAA $\neq$ Asn; NC_007438). However, this assignment in the genome of $S$. kowalevskii (AAA $=$ Lys) also occurs in other invertebrate mtDNAs.

We determined the first mitochondrial genome of a pterobranch, Rhabdopleura compacta, and compared the genome architecture and the genetic code to other deuterostome and metazoan genomes. The genome of $R$. compacta shows an unusual strand-specific bias, which strongly affected the protein sequences, and suggests an inversion of the replication direction. This precluded the phylogenetic reconstruction of Deuterostomia including Pterobranchia by protein sequence analyses. The order of protein-coding genes of $R$. compacta is similar to the hypothetical ancestral arrangement of deuterostomes but is also not informative for phylogenetic reconstruction within the Deuterostomia. The codon-anticodon distribution and the codon assignments in the pterobranch genome reject an adaptation of anticodon sequences to the most frequent codons, but give strong support for selection to an efficient tRNA translation system with 
anticodon sequences showing highest versatility. Features of its tRNA system support the grouping of Ambulacraria (Echinodermata and Hemichordata).

\section{Results and Discussion}

\section{General Features of the Mitochondrial Genome of} Rhabdopleura compacta

The mitochondrial genome of $R$. compacta comprises a 15,814 bp long circular DNA molecule with a slightly higher AT-content (66\%) than enteropneust genomes (52 - 60\%). It shows one of the strongest strand-specific biases of metazoan genomes with high amounts of Thymine (48\%) and Guanine (23\%) but low amounts of Adenine (18\%) and Cytosine (11\%) on the main-coding strand causing strong negative AT- and positive GC-skews (Figure 1). The main-coding strand bears several oligoT-stretches with lengths of six (48 times), seven (38 times), eight (19 times) and nine nucleotides (10 times), while the longest stretch of 13 T's is found only once. The large amount of Thymine leads to a higher total AT-content, although the GT-content is also markedly increased in all Rhabdopleura mt genes compared to closely related taxa (Table 1).

The genome contains all genes typical for Metazoa, i.e., 13 protein-coding, two rRNA, and 22 tRNA genes (Figure 2). All protein-coding genes start with ATG or GTG and end with a complete stop-codon with the exception of $C O X 3$ and ND4. Both genes overlap with downstream tRNA genes suggesting an incomplete termination codon "T" as described in Ojala et al. [32]. The ATP8 protein is unusual: about 20 residuals of the $\mathrm{C}$-terminus are missing. In contrast, additional parts within the protein sequences are detected within ATP6 (about 50 aa) and ND3 (about 28 aa). All protein-coding genes are located on the same strand and furthermore, the strand-specific nucleotide skews are predominantly found in protein-coding genes (Figure 1 and 2). Moreover, the oligoT-stretches are frequently located in protein-coding regions. The longest $\mathrm{T}$-stretch is located within the coding-region of $C O X 3$ leading to four phenylalanine residues in series within an evolutionary conserved phenylalanine-rich domain.

The 22 tRNA genes have been identified based on the predicted cloverleaf secondary structure. The increased GT-content has also been observed in the rRNA and, to a lesser extent, in the tRNA genes (Table 1). The two rRNA genes have been detected by alignments. Their boundaries have been recovered only for the $12 S \mathrm{rRNA}$, whereas the $16 S$ rRNA has been determined by the flanking tRNA genes. The $12 S$ rRNA is $842 \mathrm{bp}$ in size and similar to the $12 S r R N A$ of the enteropneusts $(815$ bp - $851 \mathrm{bp}$ ) showing $41-42 \%$ sequence identities. The $16 S$ rRNA ( $\leq 1,342 \mathrm{bp})$ is much shorter than the enteropneust sequences (1,498 bp - 1,504 bp) but exhibits 41 $42 \%$ sequence identity with them. The exact ends of the $16 S$ rRNA is uncertain, the gene is possibly surrounded by short UAS (unassigned sequences).

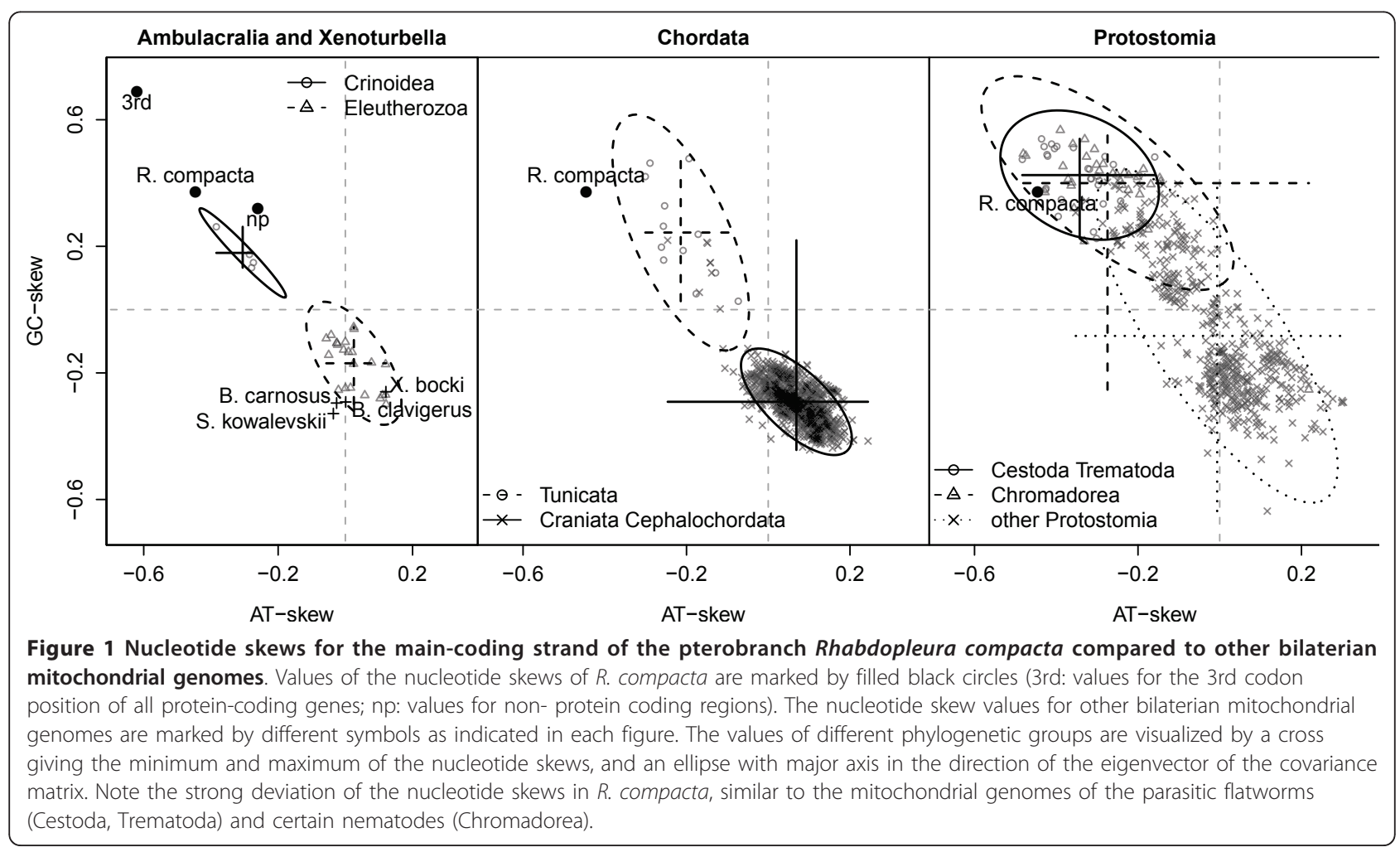


Table 1 AT- and GT-content of mtDNA genes from $R$. compacta compared to mtDNAs of Enteropneusta

\begin{tabular}{clccc}
\hline \multirow{2}{*}{ tRNA genes } & & Rhabdopleura compacta & Balanoglossus carnosus & Balanoglossus clavigerus \\
\hline & AT-content \% (total) & $65.7(964)$ & $52.6(823)$ & $53.4(832)$ \\
\cline { 2 - 5 } rRNA genes & GT-content \% (total) & $56.7(833)$ & $51.0(797)$ & $50.8(792)$ \\
\cline { 2 - 5 } & AT-content \% (total) & $68.8(1502)$ & $55.2(1276)$ & $55.4(1283)$ \\
\hline PT-content \% (total) & $64.3(1404)$ & $43.1(998)$ & $42.6(986)$ \\
\hline & AT-content \% (total) & $65.7(8012)$ & $50.5(5851)$ & $52.4(6209)$ \\
\cline { 2 - 5 } & GT-content \% (total) & $73.4(8955)$ & $45.4(5260)$ & $45.2(5359)$ \\
\hline
\end{tabular}

Surprisingly, only $1 \%$ of the genome consists of UAS and there is no large UAS region at all. The longest UAS region spans $24 \mathrm{bp}$ and is located between the ATP6 and ND6 genes (Figure 2).

\section{Strand-specific Nucleotide Biases}

Almost all metazoan mitochondrial genomes present obvious strand-specific AT- and GC-skews (Figure 1) which is usually explained by the (asymmetrical) stranddisplacement mechanisms inherent in the replication of mitogenomes [23]. Vertebrate genomes present pronounced AT- and GC-skews on the two strands having an AC-rich main-coding strand (Figure 1) (e.g. [25]). This strand-specific mutational bias is explained by the asymmetrical replication. Replication starts from two distinct replication initiation sites for the GT-rich (heavy) strand (oriH) and the AC-rich (light) strand (oriL) $[24,25]$, respectively. While the replication of the genome starts on oriH, the replication of the light strand starts later on oriL, when the replication fork from oriH has passed this point [24]. The two strands are therefore single-stranded for different lengths of time. In mammalian genomes, the oriL is located $2 / 3$ apart from the oriH exposing always the same strand as single-stranded. Further, the time of the single-stranded state increases from the oriH to the oriL showing shortest time on oriL, longest time of single-stranded on oriH and long time of single-stranded in the remaining
1/3 part [24]. The faster accumulation of Guanine and Thymine nucleotides in single-stranded DNA then leads to the observed strand-specific AT- and GC-skews [23-27].

However, investigations on mammalian mtDNAs suggest possible alternative L-strand syntheses [33-35] or additional replication modes, which might be tissue- or stage-specific [36-40]. These could substantially reduce the time of single-stranded compared to the conventional strand-displacement mode.

The strand specific accumulations of the Guanine and Thymine nucleotides in the genome of $R$. compacta show typical mutational pattern as proposed by the strand-displacement mode. In particular, the $(\mathrm{G}+\mathrm{T}) /(\mathrm{A}+\mathrm{C})$ ratios in protein-coding sequences shows a gradient along the genome of $R$. compata (Figure 2) in congruence with the conventional strand-displacement mode. The lowest $(\mathrm{G}+\mathrm{T}) /(\mathrm{A}+\mathrm{C})$ ratios are found between the genes ND5 and $C Y T B$, suggesting that the oriL is located in this region since the shortest time of single-strandedness should cause the lowest bias. The $(\mathrm{G}+\mathrm{T}) /(\mathrm{A}+\mathrm{C})$ ratios increase from $C Y T B$ to $N D 2$, while high ratios are recovered between ND2 and ND5 (Figure 2). The high ratios suggest that oriH is located close to the end of the ND2 gene. Both oriL and oriH have to be expected within genes because large unassigned sequences (UAS) that could contain regulatory elements are missing in the genome of $R$. compacta.

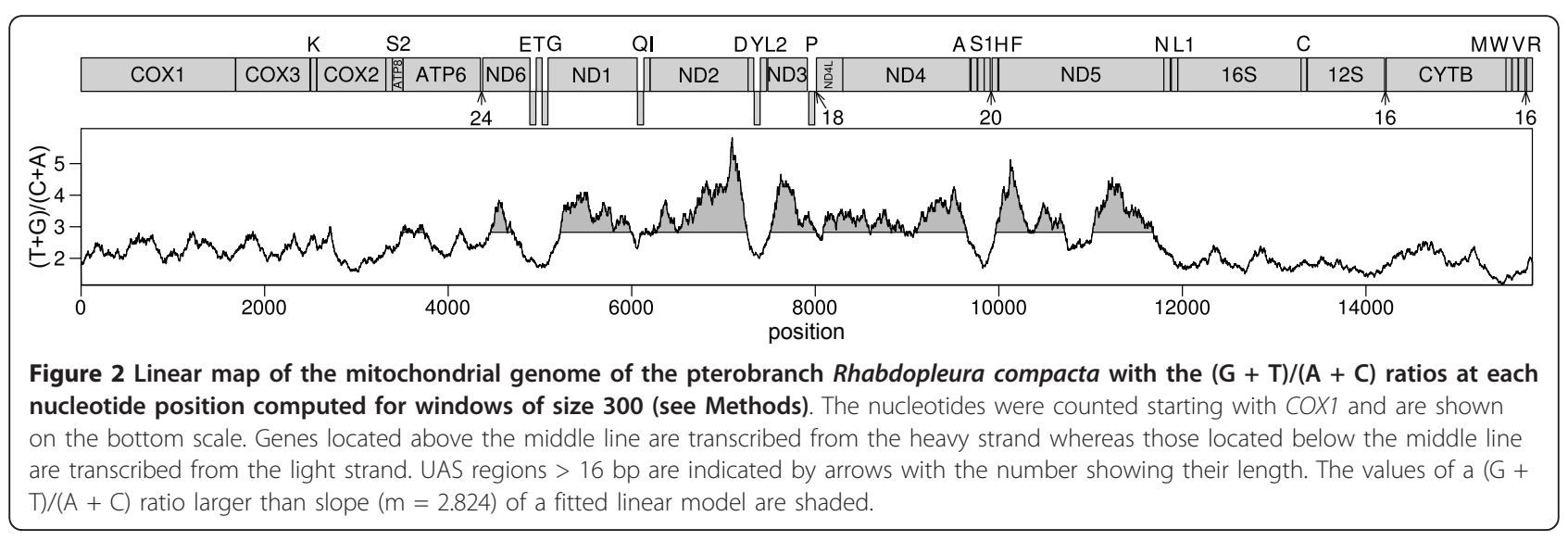


Assuming that there are competing modes of replication for metazoan mitogenomes, the extreme strand specific nucleotide bias in $R$. compacta might indicate that the strand-displacement mode is more dominant than in closely related species. However, the basal protostome clades Platyhelminthes and Chromodorea exhibit a similarly strong strand specific nucleotide bias as $R$. compacta (Figure 1), and similar replication modes might be used in these taxa.

The strand specific mutational bias in $R$. compacta is inverted on the main-coding strand compared to most other deuterostome genomes (Figure 1). This may be explained by an inversion of the direction of replication which has been described for Crinoidea [29], a subgroup of the echinoderms, and several other, unrelated taxa [23]. Only few gene inversions are found in the genome of $R$. compacta compared to genomes of Vertebrata (the ND6 and five tRNA genes) or to the enteropneust Balanoglossus (the ND6 and six tRNA genes) suggesting that the inversion of regulatory elements lead to the inverted replication direction.

\section{Genetic Code and the tRNA System}

All codons are present in the protein-coding genes of $R$. compacta, including the codons TAA and TAG as stop codons. Most assignments of the amino acid codons could be determined on conserved sites within a metazoan alignment using GenDecoder v1.6 [41], whereas five codon assignments could be recovered only with weak support (Table 2). Two of them could be well determined based on weakly conserved or variable sites: UCC (46\% Ser) and AAA (69\% Lys, no Asn) while only uncertain results were obtained for the codons AUC, UGC, and CUC. However, the codons AUC and UGC present the reverse complement of the anticodon sequence of the tRNA $\mathrm{Ale}_{\mathrm{IGAU}}$ and the RNA $_{\text {Cys } / G C A}$, which support the assignment as Ile and Cys, respectively (Table 2). The CUC codon specifies $27 \%$ as Ile, $24 \%$ as Phe, and $23 \%$ as Leu. Due to the assignment of CUC as Leu in all bilaterian genetic codes deposited in NCBI database, this assignment was also assumed for $R$. compacta (Table 2).

The codon usage in the mitochondrial genome of $R$. compacta shows a strong preference of synonymous codons ending with Thymine or Guanine (Figure 1 and Table 2), which is in contrast to most vertebrate mtDNAs [17]. Moreover, the nucleotide composition of the tRNA genes is adapted to the increased GT-content (Table 1), but most anticodons of the tRNA genes still show the typical sequence of other metazoan genomes $[19,20]$ having anticodons GNN for NNY codons, UNN for NNR codons and also UNN for four-fold degenerate codon families (Table 2). Therefore, the reverse complements of the anticodon sequences show usually the low frequent codons (Table 2) rejecting an adaptation of the anticodon sequences to most frequent codons (see [17]). In contrast, this strongly supports that an effective

Table 2 Codon usage and tRNA anticodons in the pterobranch $\boldsymbol{R}$. compacta

\begin{tabular}{|c|c|c|c|c|c|c|c|c|c|c|c|}
\hline UUU & 554 & Phe & UCU & 233 & Ser & UAU & 159 & Tyr & UGU & 99 & Cys \\
\hline UUC & 35 & Phe * & UCC & 8 & Ser & UAC & 28 & Tyr * & UGC & 10 & Cys * \\
\hline UUA & 205 & Leu * & UCA & 32 & Ser * & UAA & 2 & term & UGA & 48 & $\operatorname{Trp} *$ \\
\hline UUG & 210 & Leu & UCG & 21 & Ser & UAG & 9 & term & UGG & 89 & Trp \\
\hline CUU & 102 & Leu & CCU & 93 & Pro & CAU & 70 & His & CGU & 46 & Arg \\
\hline CUC & 5 & Leu? & CCC & 5 & Pro & CAC & 8 & His * & CGC & 4 & Arg \\
\hline CUA & 19 & Leu * & CCA & 10 & Pro * & CAA & 16 & $\mathrm{Gln} *$ & CGA & 15 & $\operatorname{Arg} *$ \\
\hline CUG & 14 & Leu & CCG & 11 & Pro & CAG & 31 & Gln & CGG & 19 & $\operatorname{Arg}$ \\
\hline
\end{tabular}

\begin{tabular}{|c|c|c|c|c|c|c|c|c|c|c|c|}
\hline AUU & 175 & Ile & $\mathrm{ACU}$ & 76 & Thr & AAU & 79 & Asn & AGU & 87 & Ser \\
\hline AUC & 9 & $\underline{\text { lle * }}$ & ACC & 3 & Thr & $A A C$ & 8 & Asn * & AGC & 10 & $\underline{\text { Ser * }}$ \\
\hline AUA & 52 & Ile & ACA & 5 & Thr * & AAA & 10 & Lys & AGA & 40 & Ser \\
\hline AUG & 96 & Met ${ }^{*}$ & ACG & 5 & Thr & AAG & 59 & Lys * & $A G G$ & 20 & Lys \\
\hline
\end{tabular}

\begin{tabular}{llllllllllll}
\hline GUU & 289 & Val & GCU & 99 & Ala & GAU & 60 & Asp & GGU & 158 & Gly \\
\hline GUC & 17 & Val & GCC & 4 & Ala & GAC & 9 & Asp * & GGC & 8 & Gly \\
\hline GUA & 39 & Val * & GCA & 12 & Ala * & GAA & 17 & Asp * & GGA & 63 & Gly * \\
\hline GUG & 64 & Val & GCG & 11 & Ala & GAG & 59 & Asp & GGG & 182 & Gly \\
\hline
\end{tabular}

The total number of codons is shown. The assignment of each codon was detected within a metazoan alignment using GenDecoder server v1.6 [41]. The reverse complements of the anticodons are marked by asterisks, deviations from the versatility rule $[15,16,18]$ are underlined. One codon (question mark) could not be determined with certainty. 
translation system is based on anticodon sequences of tRNA genes with highest versatility to all recognized codons independent of their frequency $[15,16,18]$.

Three deviations from the anticodon system with such highest versatility are found in the genome of $R$. compacta (Table 2) which are also recovered in some other deuterostome genomes and which are all correlated to codons with different assignments (Table 3).

(i) The anticodon sequence for Met is derived as it shows the reverse complement to the AUG codon instead of the AUA codon. This is found in most metazoan genomes and may be important for an effective translation of the start codon AUG (see [17]). The AUA codon shows different assignments within Deuterostomia. In all Echinodermata, the three Enteropneusta and in $R$. compacta, the AUA codon is assigned as Ile (Table 3) while it encodes Met in Chordata (Urochordata, Cephalochordata, and Vertebrata). Since no change in the anticodon of the tRNA $_{\text {Ile }}$ is found, the additional recognition of the AUA codon by the tRNA Ile should be based on other mechanisms which may have arisen in the lineage leading to the Ambulacraria.

(ii) The tRNA $A_{\text {Ser/GCU }}$ shows also no changes in the anticodon sequences within Deuterostomia and recognises the AGT and AGC (AGY) codons within highest versatility. Due to the missing of a tRNA gene for the codons AGA and AGG (AGR), these codons are partially

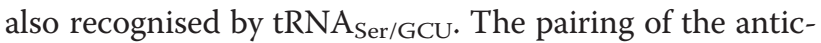
odon sequence of $\mathrm{RNA}_{\mathrm{Ser} / \mathrm{GCU}}$ to the codon AGA may be possible under special conditions; whereas the recognition of the AGG codon is assumed to be more complicated $[15,42]$. Within Deuterostomia, only the genomes of Echinodermata show obviously an assignment of Ser for all four codons. The pairing of the AGG codon to the tRNA $_{\text {Ser/GCU }}$ in echinoderms is explained by a guanosine methylation on the anticodon sequence that enables this pairing [42,43]. The genomes of Chordata and Tunicata possess a different assignment of both codons (AGG and AGA) which is based on an additional tRNA gene for Gly in Tunicata [44] and a unique pathway to recognize these codons as stop codons in Chordata [45,46]. In the genome of $R$. compacta, the codon AGA assigns Ser while the codon AGG is reassigned to Lys (Table 3).

(iii) The assignment of the codon AGG as Lys is supported for the genome of Rhabdopleura by eight highly conserved sites ( $83 \%$ with Lys) and two weakly conserved sites (63\% with Lys), but has not been detected in other deuterostome mtDNAs. However, several taxa within Arthropoda also have an assignment of AGG for Lys. All these genomes and the genome of $R$. compacta present an unusual anticodon sequence of tRNA $\mathrm{L}_{\text {Lys }}$ showing the sequence "CUU" instead of "UUU" (Table 3) [47]. Only the second codon position of the codon AGG mismatches to the anticodon sequence $t \mathrm{RNA}_{\mathrm{Lys} / \mathrm{CUU}}$ and may enable the codon reassignment to Lys. Notably, the arthropod lineage shows also mtDNAs with $\mathrm{tRNA}_{\mathrm{Lys} / \mathrm{CUU}}$ or $\mathrm{tRNA}_{\mathrm{Lys} / \mathrm{UUU}}$ but missing the reassignment. The distributions of the anticodon sequences within arthropods suggest an ancestral mutation in the tRNA $A_{\text {Lys }}$ but could not support (but also not reject) an ancestral assignment of AGG as Lys [47]. Therefore, several independent backmutations to the usual anticodon sequence "UUU" and several reassignments of AGG to Lys may have occurred [47]. Similar to this, the genomes of Echinodermata and the enteropneust Balanoglossus spp. have also the derive anticodon sequence "CUU" while the genome of the enteropneust Saccoglossus shows the usual anticodon sequence of "UUU" (Table 3). All these genomes lack the reassignment of AGG to Lys. Furthermore, the translation efficiency for the codon AAA by the $\mathrm{RNA}_{\mathrm{Lys} / \mathrm{CUU}}$

Table 3 Mitochondrial Genetic Code of Deuterostomia and selected Protostomia

\begin{tabular}{|c|c|c|c|c|c|c|c|}
\hline & $\begin{array}{l}\text { Codon } \\
\text { AUA }\end{array}$ & $\begin{array}{l}\text { Codon } \\
\text { AAA }\end{array}$ & $\begin{array}{l}\text { Codon } \\
\text { AGG }\end{array}$ & $\begin{array}{l}\text { Codon } \\
\text { AGA }\end{array}$ & $\begin{array}{l}\text { Anticodon loop of } \\
\text { tRNA }_{\text {Asn }}\end{array}$ & $\begin{array}{l}\text { Anticodon loop of } \\
\text { tRNA }_{\text {Lys }}\end{array}$ & $\begin{array}{l}\text { Source/ } \\
\text { CodTab }\end{array}$ \\
\hline Rhabdopleura compacta & Ile & Lys & Lys & Ser & $\cup \cup \underline{G U U} A C$ & $C \cup \underline{C \cup U} A A$ & $\begin{array}{l}\text { this study, table } \\
1\end{array}$ \\
\hline Balanoglossus spp. & He & - & - & Ser & CU GUU AA & $C \cup \underline{C \cup U} A A$ & {$[30,31]$} \\
\hline Saccoglossus kowalevskii & lle & Lys & - & Ser & $C \cup \underline{G \cup U} A A$ & $C \cup \underline{U U} \mathrm{AA}$ & NC_007438 \\
\hline Echinodermata & He & Asn & Ser & Ser & $C C \underline{G \cup U} \mathrm{AA}$ & $C \cup \underline{C \cup U} A A$ & CodTab 9 \\
\hline Tunicata & Met & Lys & Gly & Gly & CU GUU AA & $C \cup \underline{\cup U U} \mathrm{AA}$ & CodTab13 \\
\hline Vertebrata & Met & Lys & term & term & $\mathrm{CU} \underline{\mathrm{GUU}} \mathrm{AA}$ & $C \cup \underline{\cup \cup U A}$ & CodTab 2 \\
\hline Cephalochordata & Met & Lys & - & Ser & $C \cup \underline{G \cup U} A A$ & $C \cup \underline{\cup U} A A$ & CodTab 5 \\
\hline invertebrates & Met & Lys & Ser & Ser & & $C \cup \cup \cup \cup A A$ & CodTab 5 \\
\hline $\begin{array}{l}\text { hypothetical ancestral state in } \\
\text { Arthropoda }\end{array}$ & Met & Lys & Lys/Ser & Ser & & $C \cup \underline{C \cup U} A A$ & {$[47]$} \\
\hline
\end{tabular}

The variable codon assignments within mtDNA of Deuterostomia and selected anticodon loop sequences (the anticodon sequences are underlined) are shown. The entire genetic codes available at the web site http://www.ncbi.nlm.nih.gov/Taxonomy/Utils/wprintgc.cgi are given by the codon table number (CodTab). Amino acids are shown in the 3-letters-abbreviations. Low frequent codons are given by cursive amino acid abbreviations while unpredictable or completely missing codons are shown by dash. 
could be reduced due to deviation from the highest versatility in the $3^{\text {rd }}$ codon site (anticodon sequence changed to " $C$ " instead of " $U$ " in the first site) and may have caused the lack of the AAA codons in the genomes of Balanoglossus [30] as well as the reassignment of the codon AAA to Asn in echinoderms [48]. The recognition of the codon AAA by the $\mathrm{TRNA}_{\mathrm{Asn} / \mathrm{GUU}}$ in echinoderm genomes may be based on a further mutation on position 33 in the anticodon loop of the tRNA $\mathrm{Asn}_{\text {isn }}[31,48]$ which is missing in pterobranch and enteropneust genomes (Table 3).

In summary, the distribution of the tRNA $\mathrm{L}_{\mathrm{Lys}}$ anticodon sequence within Deuterostomia and the reassignments of the codon AAA (in Echinodermata) and AGG (R. compacta) suggest a $\mathrm{RNA}_{\mathrm{Lys} / \mathrm{CUU}}$ as an apomorphic character for the taxon Ambulacraria (Table 3). Furthermore, our data support the codon reassignments as a two-step-process: The first step was the reduced recognition in mtDNAs of Ambulacraria as a consequence of a mutation in $\mathrm{tRNA}_{\mathrm{Lys} / \mathrm{CUU}}$ abandoning AAA and the lack of a suitable tRNA for codon AGG. These reductions paved the way for adaptations in two other tRNAs to recognize these codons: AGG by tRNA $\mathrm{Lys}_{\mathrm{LUU}}$ in $R$. compacta and AAA by tRNA Asn/GUU $_{\text {in Echinodermata. Both }}$ steps were forced by an effective translation system in congruence to the "Modified Ambiguous Intermediate Theory of Codon Reassignment" [49-51]. The alternative "Codon Capture Hypothesis of Codon Reassignment" [52] assumes mutational pressure in the first step leading to codon losses in the genome. This seems to be unlikely because the genome of $R$. compacta presents a strong mutational bias in all protein-coding genes, but no codon is missing.

\section{Protein-Coding Genes}

The GT nucleotide composition has an important influence on the protein composition of nuclear genomes [53]. Similarly, the mutational bias in the genome of $R$. compacta strongly influences the protein sequences, even if highest GT-bias is detected in the third codon position (Figure 1). The GT-rich main-coding strand of $R$. compacta exhibits all protein-coding genes showing an increase of GT-rich codons at the expense of AC-rich codons. This leads to unusual amino acid composition in all proteins (Figure 3). The abundance is increased for Phe, Gly, Val, Trp which all are encoded by exclusively GT-rich codons (Figure 3). In contrast, the amino acids Thr, Pro, Asn, His, and Gln are decoded solely by ACrich codons and are decreased in their abundances in the proteins of $R$. compacta compared to the proteins of closely related species (Figure 3 ).

This strong bias within the amino acid sequence hampered the phylogenetic analyses of the protein-coding genes leading to extreme long branch lengths (Figure 4).

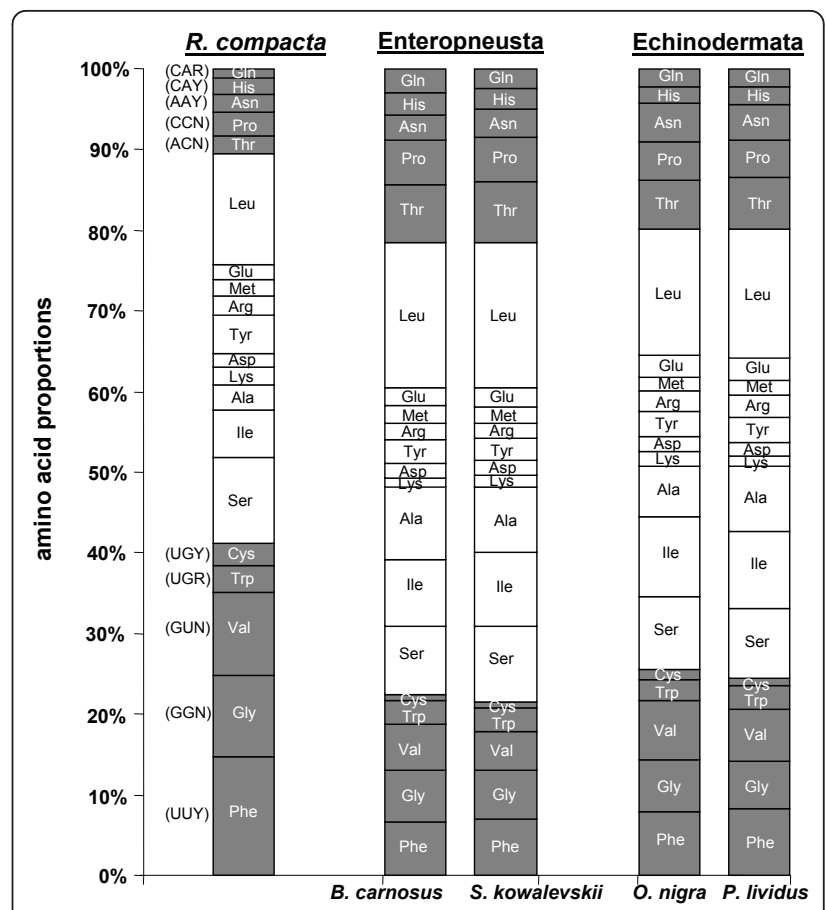

Figure 3 Amino acid composition of the 13 protein-coding genes in Rhabdopleura compacta compared to closely related deuterostome species: the enteropneusts Balanoglossus carnosus and Saccoglossus kowalevskii, the sea urchin Paracentrotus lividus and the brittle star Ophiocomina nigra. Amino acids solely encoded by GT or AC rich codons are indicated by white letters. These codons are listed on the left and are identical for all mtDNAs of Deuterostomia.

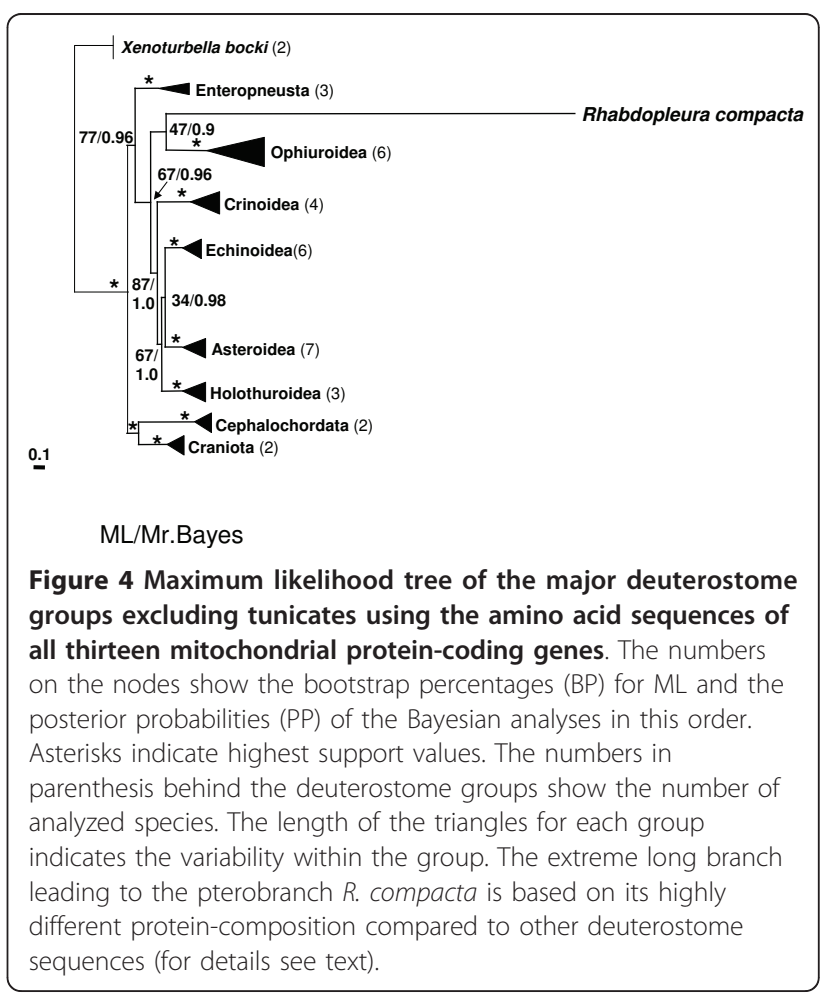


Both ML and the Bayesian approaches resulted in a meaningless sistergroup relationship of $R$. compacta to the echinoderm subgroup Ophiuroidea, hence rejecting a monophyletic Echinodermata and Hemichordata (Figure 4). While the monophyly of Echinodermata is well supported by several other analyses [6], the mitochondrial genomes of Ophiuroidea show an accelerated evolutionary rate $[11,54,55]$. Thus, the grouping of $R$. compacta and Ophiuroidea most likely is caused by a long branch artefact due to the unusual protein composition. However, the protein composition of $R$. compacta suggests a long time of adaptive evolution under the same mutational pressure.

\section{Gene Order Evolution}

The mtDNAs of most deuterostome groups show similar gene arrangements, especially the order of the proteincoding genes. The consensus gene order of vertebrate genomes is assumed to represent the ancestral arrangement for Deuterostomia, if not for Metazoa [8]. Only the genomes of Tunicata show unique gene arrangements (including two additionally tRNA genes) featuring all genes on one strand and having unusual high rearrangement rates within the group (e.g. [13]). The mitochondrial genome of $R$. compacta presents a new gene arrangement showing high differences in the tRNA and rRNA gene order compared to the assumed ancestral deuterostome as well as to the known hemichordate arrangements (Additional file 1, Figure S1).

With regard to the order of the protein-coding genes the arrangement of Balanoglossus (identical to Xenturbella bocki) appears to represent the ancestral state within Hemichordata, since only one translocation of CYTB or ND6 from the vertebrate/cephalochordate arrangement has to be hypothesized (Figure 5).

Within Hemichordata, two rearrangements are necessary to interconvert the basal Balanoglossus gene order into that of Saccoglossus kovalewskii (the inversion of $C O X 1$, and the transposition of either ND1 or ND2). The gene order of $R$. compacta needs three steps to be derived from the ancestral Balanoglossus order (inversion of ND6, translocation of $\mathrm{COX} 3$, and transposition of the fragment containing ND1 and ND2) (Figure 5). However, these rearrangements have to be addressed as autapomorphies and thus, no synapomorphic characters could be detected in gene order evolution within Hemichordata, which would allow phylogenetic reconstruction within the group.

Notably, the gene order of $R$. compacta can be derived directly from the assumed basal deuterostome arrangement (Vertebrata) by the same number of three rearrangements, which however would render Hemichordata paraphyletic (Figure 5).
Although the ancestral arrangement of the fragment containing ND1 and ND2 cannot be surely determined [11], the echinoderm genomes show an apomorphic translocation of the ND4L gene, which is not recovered in any other deuterostome genome (Figure 5).

\section{Conclusion}

The unusual mitochondrial genome of $R$. compacta features a strong, reversed asymmetrical mutational constraint compared to most other deuterostome genomes. This may have arisen by an inversion of the replication direction in the lineage leading to $R$. compacta and by an adaptation to this bias in the protein sequences. Due to this bias, phylogenetic analyses of protein-coding genes failed. However, the tRNA system supports the monophyly of Ambulacraria, comprising Hemichordata and Echinodermata by the synapomorphic characters $\mathrm{RNA}_{\mathrm{Lys} / \mathrm{CUU}}$ and the assignment of ATA as Ile. The strong strand-specific mutational bias in the genome of $R$. compacta allows further important insights in the evolution of the tRNA system. The observed codon usage and their anticodon sequences strongly support a selection pressure to an efficient translation system possessing anticodon sequences which pair most easily with all recognized codons, independent of their frequency (anticodons with highest versatility) similar to most tRNA genes of fungal mtDNAs [15]. Mutations in the anticodon sequence changing the recognition efficiency could lead to a bias in the codon distributions and could cause the deletion of codons from the genome. Furthermore, similar mutations in the anticodon sequence could cause similar adaption processes in the tRNA translation system as found in the convergent assignment of AGG as Lys in the genome of $R$. compacta and some arthropod genomes. Our analyses support the disappearance and reassignment of codons in response to a selection pressure for an efficient translation system as described in the "Modified Ambiguous Intermediate Theory of Codon Reassignment” [49-51].

\section{Methods \\ DNA Extraction, Amplification, Sequencing and Annotation}

About 50 adult zooids of Rhabdopleura compacta Hincks, 1880 were dissected from their coenecia, which were attached to disarticulated shells of Glycymeris glycymeris, dredged off from Stoke Point (near Plymouth, UK, 50 $\left.17^{\prime} \mathrm{N}, 4^{\circ} 01^{\prime} \mathrm{W}\right)$ in Mai 2006 from a depth of 21-24 m. Genomic DNA was extracted using phenol - chloroform extraction, following proteinase $\mathrm{K}$ digestion. The complete mitochondrial genome was amplified using two overlapping fragments. The first fragment was amplified with the specific primer pair GTGGTGGAGTACCCTTTTAAGACTG and GACCCAATAGTTGAAGCATGATGCC, 


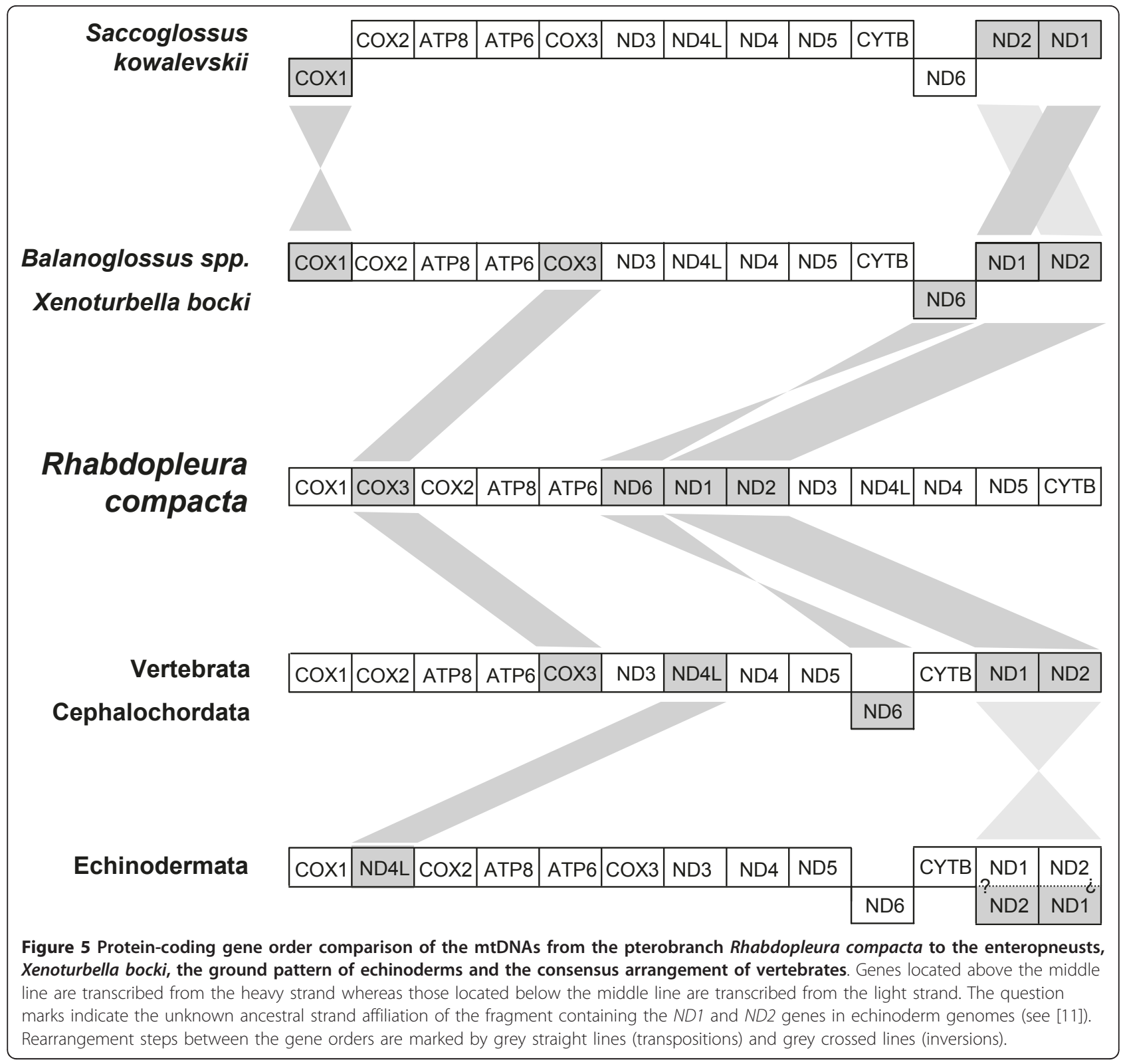

which was determined from the COX1 sequences that we found in our Rhabdopleura EST (M. Perseke et al., unpublished). The second fragment was amplified with the specific primer pair GTTTACTTTGGGGGGTTGCACTGG and CCAACGCTCAATCAGCTTCAAGAGC, which was designed based on the first COX1 fragment sequenced. The PCR reactions were performed with a Mastercycler machine (Eppendorf AG, Hamburg, Germany). The cycling was set up with an initial denaturing step at $94^{\circ} \mathrm{C}$ for 3 minutes, followed by 35 cycles of denaturing at $94^{\circ} \mathrm{C}$ for 60 seconds, annealing at $60^{\circ} \mathrm{C}$ for 30 seconds and elongation at $68^{\circ} \mathrm{C}$ for 5 minutes.

The $25 \mu \mathrm{l}$ PCR reaction amounted $0.3 \mathrm{U}$ of the Phusion High-Fidelity DNA polymerase (Finnzymes, Espoo,
Finland) and 0.3 ng DNA as well as $5 \mu \mathrm{l} 5 \times$ Phusion GC buffer (Finnzymes), $2.5 \mu \mathrm{l} \mathrm{dNTP} \mathrm{mix} \mathrm{(10} \mathrm{mM} \mathrm{each)} \mathrm{and}$ $1 \mu \mathrm{l}$ of forward and reverse primers, respectively $(10 \mu \mathrm{M}$ each). The PCR product of the large fragment was sequenced by primer walking as described by Perseke et al. [54]. Additional starting points for faster primer walking were obtained by preparing a "mini" DNA library using four-cutter restriction enzymes and the pGem-T plasmid Vector (Promega). All PCR products were sequenced directly on an ABI 3100 automated sequencer (Applied Biosystems) using the BigDye Termination v3.1 Cycle Sequencing Kit (Applied Biosystems). After concatenating the sequences to the complete genome, all protein-coding genes and the rRNA genes were identified by 
alignments. All tRNA genes were identified by using the tRNAscan-SE 1.23 server [56]. The entire mitochondrial DNA sequence was submitted to the GenBank under the accession number FN908482.

Analysis of Nucleotide Composition and the Genetic Code The strand biases of the main-coding strand, given as the AT-skew $[(\mathrm{A}-\mathrm{T}) /(\mathrm{A}+\mathrm{T})]$ and the GC-skew $[(\mathrm{G}-\mathrm{C}) /$ $(\mathrm{G}+\mathrm{C})$ ] [57], were calculated using an automatic tool developed for this purpose for both the complete genome of $R$. compacta and all complete mitogenomes available in NCBI RefSeq 41 [58]. In addition, the ATand GC-skews were computed separately excluding protein-coding sequences and further using only the $3^{\text {rd }}$ codon positions of the protein-coding genes of $R$. compacta.

The values of the $(T+G) /(C+A)$ ratios were determined for each position in the genome of $R$. compacta using a window starting 149 positions to the left and ending 150 positions to the right.

The genetic code of the $R$. compacta genome was predicted by evaluating four different degrees of conserved codons (highly conserved, conserved, weakly conserved and variable sites) in the alignment of 56 metazoan sequences implemented in the GenDecoder server v1.6 [41].

\section{Analysis of Gene Order}

The genome rearrangements were studied using the webbased CREx software [59]. The gene order of $R$. compacta was compared to the following deuterostome genomes: both Balanoglossus species [11,30]; Saccoglossus kowalevskii (NC_007438), Xenoturbella bocki $[10,14]$, the putative ancestral arrangement in Echinodermata [11], the consensus gene order of Vertebrata [12], the ancestral order of Cephalochordata [9] and all known gene orders of Tunicata [13]. Further, the gene order was compared to Platyhelminthes [60], and Nematoda [61]. All comparisons were performed twice - with and without - the tRNA and rRNA genes.

\section{Phylogenetic Analyses of Protein-Coding Sequences}

The phylogenetic analyses of the protein-coding genes were carried out by adding the $R$. compacta protein sequences to the taxon set "Deuterostomia" from Perseke et al. [11]. All protein-coding genes were aligned separately using the $\mathrm{T}$-Coffee server with the default parameter set [62]. The sequences were then truncated, so that no genes started and ended with a gap. Finally, they were concatenated. The "mtRev" model of amino acids was selected and the gamma shape parameter and the proportion of invariable sites were determined using PhyML v.2.4.4 [63]. The Maximum Likelihood (ML) analyses were carried out with PhyML v.2.4.4 [63] using the determined parameters and six categories of substitution rates. The robustness of bifurcations was estimated with bootstrap analyses (100 replicates). The Bayesian analyses were performed with MrBayes v.3.1.2 [64] using the determined parameters and six categories of substitution rates. The calculation was run for 1,000,000 generations, with a sampling frequency of 10 generations and burn-in of the first 25,000 trees. The remaining trees were tested for stability of the likelihood values and used to compute the posterior probabilities.

\section{Additional material}

Additional file 1: Figure S1 - Gene order comparison of the mtDNAs from the pterobranch Rhabdopleura compacta to the enteropneust genomes. Genes located above the middle line are transcribed from the heavy strand whereas those located below the middle line are transcribed from the light strand. Grey regions highlight conserved protein-coding gene arrangements. The black lines show the tRNA rearrangements (transposition, inversion and reverse transposition) within the conserved blocks.

\section{Acknowledgements}

We thank John D. D. Bishop and Christine Wood from the Marine Biological Association of the United Kingdom and Atsuko Sato from the University of Oxford for providing us with colonies from $R$. compacta, laboratory equipment for DNA extraction and their helpful assistance to collect zooids. Many thanks to Georg Mayer for helpful discussions of our manuscript. This work was supported by the DFG grants SCHL 229/14-1 and STA 850/3-1 as part of the SPP-1174 (Deep Metazoan Phylogeny).

\section{Author details}

${ }^{1}$ Molecular Evolution and Animal Systematics, University of Leipzig, Talstr. 33, 04103 Leipzig, Germany. 'Laboratory of Marine Biology, South China Sea Institute of Oceanology, Chinese Academy of Science, 164 West Xingang Road, 510301 Guangzhou, PR China. ${ }^{3}$ Parallel Computing and Complex Systems Group, University of Leipzig, Johannisgasse, 26, 04103 Leipzig, Germany. ${ }^{4}$ Bioinformatics Group, Dept. of Computer Science, University of Leipzig, Härtelstr, 16-18, 04107 Leipzig, Germany. ${ }^{5}$ Interdisciplinary Center for Bioinformatics, University of Leipzig, Härtelstr, 16-18, 04107 Leipzig, Germany. ${ }^{6}$ Max Planck Institute for Mathematics in the Sciences, Inselstrasse 22, 04103 Leipzig, Germany. ${ }^{7}$ Fraunhofer Institut für Zelltherapie und Immunologie, IZI, Perlickstrasse 1, 04103 Leipzig, Germany. ${ }^{8}$ Center for non-coding RNA in Technology and Health, University of Copenhagen, Grønnegårdsvej 3, 1870 Frederiksberg C, Denmark. ' Department of Theoretical Chemistry, University of Vienna, Währingerstrasse 17, 1090 Wien, Austria. ${ }^{10}$ Santa Fe Institute, 1399 Hyde Park Rd, Santa Fe, NM 87501, USA.

\section{Authors' contributions}

This study was carried out in collaboration between the working groups of MS and PFS. DB, MS and PFS defined the research theme. MP, MS and DB designed methods and experiments. MP collected specimens and extracted DNA. MP and JH amplified, sequenced and analyzed the mt genome. MB calculated and displayed the nucleotide biases. MP interpreted the results and PFS, MS and DB discussed the analyses, interpretation, and presentation. All authors have contributed to the manuscript and approved the submitted version.

Received: 7 March 2011 Accepted: 20 May 2011 Published: 20 May 2011

\section{References}

1. Benito J, Pardos F: Hemichordata. In Microscopic Anatomy of Invertebrates. Volume 15. Edited by: Harrison MA. Wiley Liss. New York; 1997:15-101.

2. Sato T: Vorläufige Mitteilungen über Atubaria heterolopba gen. nov. sp. nov., einen in freiem Zustand aufgefundenen Pterobranchier aus dem Stillen Ozean. Zool Anz 1936, 115:97-106. 
3. Hyman LH: Smaller Coelomate Groups: Hemichordata. In The Invertebrates. Volume 5. New York: McGraw-Hill Book Company; 1959:72-207.

4. Halanych KM: The phylogenetic position of the pterobranch hemichordates based on 18S rDNA sequence data. Mol Phyl Evol 1995, 4:72-76.

5. Cameron CB, Garey JR, Swalla BJ: Evolution of the chordate body plan: New insights from phylogenetic analyses of deuterostome phyla. Proc Natl Acad Sci USA 2000, 97:4469-4474.

6. Winchell CJ, Sullivan J, Cameron CB, Swalla BJ, Mallatt J: Evaluating hypotheses of deuterostome phylogeny and chordate evolution with new LSU and SSU ribosomal DNA data. Mol Biol Evol 2002, 19:762-776.

7. Metschnikoff VE: Über die systematische Stellung von Balanoglossus. Zool Anz 1881, 4:139-157.

8. Lavrov DV, Lang BF: Poriferan mtDNA and animal phylogeny based on mitochondrial gene arrangements. Systematic Biol 2005, 54:651-659.

9. Nohara M, Nishida M, Miya M, Nishikawa T: Evolution of the mitochondrial genome in cephalochordata as inferred from complete nucleotide sequences from two Epigonichthys species. J Mol Biol 2005, 60:526-537.

10. Perseke M, Hankeln T, Weich B, Fritzsch G, Stadler PF, Israelsson O, Bernhard D, Schlegel M: The mitochondrial DNA of Xenoturbella bocki: genomic architecture and phylogenetic analysis. Theory of Biosci 2007 126:35-42.

11. Perseke M, Bernhard D, Fritzsch G, Brümmer F, Stadler PF, Schlegel M: Mitochondrial genome evolution in Ophiuroides, Echinoidea, and Holthuroidea: Insights in phylogenetic relationships of Echinodermata. Mol Phyl Evol 2010, 56(1):201-211

12. Boore JL: Animal mitochondrial genomes. Nucleic Acids Res 1999 27:1767-1780.

13. Stach T, Braband A, Podsiadlowski L: Erosion of phylogenetic signal in tunicate mitochondrial genomes on different levels of analysis. Mol Phylogenet Evol 2010, 55:860-870.

14. Bourlat SJ, Rota-Stabelli O, Lanfear R, Telford MJ: The mitochondrial genome structure of Xenoturbella bocki (phylum Xenoturbellida) is ancestral within the deuterostomes. BMC Evol Biol 2009, 9:107.

15. Carullo M, Xia X: An Extensive Study of Mutation and Selection on the Wobble Nucleotide in tRNA Anticodons in Fungal Mitochondrial Genomes. J Mol Evol 2008, 66:484-493.

16. Xia $X$ : The cost of wobble translation in fungal mitochondrial genomes: integration of two traditional hypotheses. BMC Evol Biol 2008, 8:211.

17. Xia X: Mutation and selection on the anticodon of tRNA genes in vertebrate mitochondrial genomes. Gene 2005, 345:13-20.

18. Tong KL, Wong JT: Anticodon and wobble evolution. Gene 2004 333:169-177.

19. Yokobori S, Suzuki T, Watanabe K: Genetic code variations in mitochondria: tRNA as a major determinant of genetic code plasticity. J Mol Evol 2001, 53:314-326.

20. Yokoyama S, Nishimura S: Modified nucleotides and codon recognition. In tRNA: Structure, Biosynthesis and Function. Edited by: Soll D, RajBhandary U. Washington, MA: ASM Press; 1995:207-223.

21. Bulmer M: The selection-mutation-drift theory of synonymous codon usage. Genetics 1991, 129:897-907.

22. Bulmer M: Coevolution of codon usage and transfer RNA abundance Nature 1987, 325(6106):728-730.

23. Hassanin A, Léger $N$, Deutsch J: Evidence for multiple reversals of asymmetric mutational constraints during the evolution of the mitochondrial genome of Metazoa, and consequences for phylogenetic inferences. Syst Biol 2005, 54:277-298.

24. Krishnan NM, Seligmann H, Raina SZ, Pollock DD: Detecting gradients of asymmetry in site-specific substitutions in mitochondrial genomes. DNA Cell Biol 2004, 23:707-714

25. Reyes A, Gissi C, Pesole G, Saccone C: Asymmetrical directional mutation pressure in the mitochondrial genome of mammals. Mol Biol Evol 1998, 15:957-966.

26. Grigoriev A: Analyzing genomes with cumulative skew diagrams. Nucleic Acids Res 1998, 26:2286-2290.

27. Francino MP, Ochman H: Strand asymmetries in DNA evolution. Trends Genet 1997, 13:240-245.

28. Frederico LA, Kunkel TA, Shaw BR: A sensitive genetic assay for the detection of cytosine deamination: determination of rate constants and the activation energy. Biochemistry 1990, 29:2532-2537.
29. Scouras A, Smith JM: The complete mitochondrial genomes of the sea lily Gymnocrinus richeri and the feather star Phanogenia gracilis: signature nucleotide bias and unique nad4L gene rearrangement within crinoids. Mol Phylogenet Evol 2006, 39:323-334.

30. Castresana J, Feldmaier-Fuchs G, Yokobori S, Satoh N, Päabo S: The mitochondrial genome of the hemichordata Balanoglossus carnosus and the evolution of deuterostome mitochondria. Genetics 1998 150:1115-1123.

31. Castresana J, Feldmaier-Fuchs G, Päabo S: Codon reassignment and amino acid composition in hemichordate mitochondria. Proc Natl Acad Sci USA 1998, 95:3703-3707.

32. Ojala D, Montoya DJ, Attardi G: tRNA punctuation model of RNA processing in human mitochondria. Nature 1981, 290:470-474

33. Brown TA, Cecconi C, Tkachuk AN: Replication of mitochondrial DNA occurs by strand displacement with alternative light-strand origins, not via a strand-coupled mechanism. Genes Dev 2005, 19:2466-2476.

34. Pikó L, Bulpitt KJ, Meyer R: Structural and replicative forms of mitochondrial DNA in tissues from adult and senescent $B A L B / c$ mice and Fischer 344 rats. Mech. Ageing Dev 1984, 26:113-131.

35. Koike K, Wolstenholme DR: Evidence for discontinuous replication of circular mitochondrial DNA molecules from Novikoff rat ascites hepatoma cells. J. Cell Biol 1974, 61:14-25.

36. Holt IJ, Jacobs HT: Response: The mitochondrial DNA replication bubble has not burst. Trends Biochem. Sci 2003, 28:355-356.

37. Yang MY, Bowmaker $M$, Reyes $A$, Vergani $L$, Angeli $P$, Gringeri $E$, Jacobs $H T$, Holt IJ: Biased incorporation of ribonucleotides on the mitochondrial Lstrand accounts for apparent strand-asymmetric DNA replication. Cell 2002, 111:495-505

38. Holt IJ, Lorimer HE, Jacobs HT: Coupled leading and lagging-strand synthesis of mammalian mitochondrial DNA. Cell 2000, 100:515-524

39. Yasukawa T, Yang MY, Jacobs HT, Holt IJ: A bidirectional origin of replication maps to the major noncoding region of human mitochondrial DNA. Mol. Cell 2005, 18:651-662.

40. Bowmaker M, Yang MY, Yasukawa T, Reyes A, Jacobs HT, Huberman JA, Holt IJ: Mammalian mitochondrial DNA replicates bidirectionally from an initiation zone. J. Biol. Chem 2003, 278:50961-50969.

41. Abascal F, Zardoya R, Posada D: GenDecoder: genetic code prediction for metazoan mitochondria. Nucleic Acids Res 2006, 34:389-393.

42. Matsuyama S, Ueda T, Crain PF, McCloskey JA, Watanabe K: A novel wobble rule found in starfish mitochondria. J Biol Chem 1998, 273:3363-3368

43. Tomita K, Ueda T, Watanabe K: 7-Methylguanosine at the anticodon wobble position of squid mitochondrial tRNA(Ser)GCU: Molecular basis for assignment of AGA/AGG codons as serine in invertebrate mitochondria. Biochim Biophys Acta 1998, 1399:78-82.

44. Kondow A, Suzuki T, Yokobori S, Ueda T, Watanabe K: An extra tRNAGly $(U * \mathrm{CU})$ found in ascidian mitochondria responsible for decoding nonuniversal codons AGA/AGG as glycine. Nucleic Acids Res 1999, 27:2554-2559.

45. Ivanov $V$, Beniaminov A, Mikheyev A, Minyat E: A mechanism for stop codon recognition by the ribosome: $\mathrm{A}$ bioinformatic approach. RNA 2001, 7:1683-1692.

46. Osawa S, Jukes ST, Watanabe K, Muto A: Recent evidence for evolution of the genetic code. Microbiol Rev 1992, 56:229-264.

47. Abascal F, Posada D, Knight RD, Zardoya R: Parallel evolution of the genetic code in arthropod mitochondrial genomes. PLOS Biol 2006, 4 e127.

48. Asakawa S, Himeno H, Miura K, Watanabe K: Nucleotide sequence and gene organization of the starfish Asterina pectinifera mitochondrial genome. Genetics 1995, 140:1047-1060.

49. Schultz DW, Yarus M: Transfer RNA mutation and the malleability of the genetic code. J Mol Biol 1994, 235:1377-1380.

50. Schultz DW, Yarus M: On malleability in the genetic code. J Mol Evol 1996, 42:597-601.

51. Massey SE, Moura G, Beltrao P, Almeida R, Garey JR, Tuite MF, Santos MAS: Comparative evolutionary genomics unveils the molecular mechanism of reassignment of the CTG codon in Candida spp. Genome Res 2003, 13:544-557.

52. Jukes TH, Osawa S: Further Comments on Codon Reassignment. J Mol Evol 1997, 45:1-8. 
53. Zhang Z, Yu J: Modeling compositional dynamics based on GC and purine contents of protein-coding sequences. Biology Direct 2010, 5:63.

54. Perseke M, Fritzsch G, Ramsch K, Bernt M, Merkle D, Middendorf M, Bernhard D, Stadler PF, Schlegel M: Evolution of mitochondrial gene orders in echinoderms. Mol Phylogenet Evol 2008, 47:855-864.

55. Scouras A, Beckenbach K, Arndt A, Smith JM: Complete mitochondrial genome DNA sequence for two ophiuroids and a holothuroid: the utility of protein gene sequence and gene maps in the analyses of deep deuterostome phylogeny. Mol. Phylogenet. Evol 2004, 31:50-65.

56. Lowe TM, Eddy SR: tRNAscan-SE: a program for improved detection of transfer RNA genes in genomic sequence. Nucleic Acids Res 1997, 25:955-964.

57. Lobry JR: Properties of a general model of DNA evolution under nostrand bias conditions. J Mol Evol 1995, 40:326-330.

58. Pruitt KD, Tatusova T, Maglott DR: NCBI reference sequences (RefSeq): a curated non-redundant sequence database of genomes, transcripts and proteins. Nucleic Acids Res 2007, 35:D61-D65.

59. Bernt M, Merkle D, Ramsch K, Fritzsch G, Perseke M, Bernhard D, Schlegel M, Stadler PF, Middendorf M: CREx: inferring genomic rearrangements based on common intervals. Bioinformatics 2007, 23(21):2957-2958.

60. Park JK, Kim KH, Kang SH, Kim JH, Eom KS, Littlewood DTJ: A common origin of complex life cycles in parasitic flatworms: evidence from the complete mitochondrial genome of Microcotyle sebastis. BMC Evol Biol 2007, 7:11

61. He Y, Jones J, Armstrong M, Lamberti F, Moens M: The mitochondrial genome of Xiphinema americanum sensu stricto (Nematoda: enoplea): Considerable economization in the length and structural features of encoded genes. J Mol Evol 2005, 61:819-833.

62. Notredame C, Higgins D, Heringa J: T-Coffee: a novel method for multiple sequence alignments. J Mol Biol 2000, 302:205-217.

63. Guindon S, Gascuel O: A simple, fast and accurate method to estimate large phylogenies by maximum-likelihood. Syst Biol 2003, 52:696-704.

64. Ronquist F, Huelsenbeck JP: MrBayes 3: Bayesian phylogenetic inference under mixed models. Bioinformatics 2003, 19:1572-1574

doi:10.1186/1471-2148-11-134

Cite this article as: Perseke et al:: The enigmatic mitochondrial genome of Rhabdopleura compacta (Pterobranchia) reveals insights into selection of an efficient tRNA system and supports monophyly of Ambulacraria. BMC Evolutionary Biology 2011 11:134

\section{Submit your next manuscript to BioMed Central and take full advantage of:}

- Convenient online submission

- Thorough peer review

- No space constraints or color figure charges

- Immediate publication on acceptance

- Inclusion in PubMed, CAS, Scopus and Google Scholar

- Research which is freely available for redistribution

Submit your manuscript at www.biomedcentral.com/submit
Biomed Central 